\title{
TOWARDS SCALABLE COLLABORATIVE LEARNING FLOW PATTERN ORCHESTRATION TECHNOLOGIES
}

\author{
Kalpani Manathunga, Davinia Hernández-Leo \\ ICT Department, Universitat Pompeu Fabra (SPAIN)
}

\begin{abstract}
Collaborative Learning Flow Patterns (CLFPs) structure learning flows to shape desired social interactions among learners leading to fruitful learning gains. It is worthwhile to study the possibilities of CLFP extensions to be applicable in large class contexts and also in Massive Open Online Courses (MOOCs) considering their dynamic, unpredictable nature. This study considers most commonly used patterns for the adaptability in such contexts from different dimensions like pedagogical interest, scalability and other related perspectives. As a result derived from the analysis, a collection of use cases is elaborated illustrating potential collaborative learning opportunities, design requirements, initial screen designs of such activities and expected functionality descriptions for novel CSCL orchestration technologies. One of these use cases is implemented in the PyramidApp tool.
\end{abstract}

Keywords: Collaborative Learning, Computer-Supported Collaborative Learning, Collaborative Learning Flow Patterns, Learning at Scale, MOOC.

\section{INTRODUCTION}

Collaborative Learning is the pedagogical approach that considers social interactions as key means to trigger rich learning processes [1]. Computer Supported Collaborative Learning (CSCL) is the research field that studies and proposes technological support to real-time management (i.e., orchestration) of learners when forming groups, allocating roles or activity resources and changing activity phases [2]. CSCL macro-scripts describe the ways and means of groups and individuals engage in interrelated collaborative activities [3], [4]. Since modelling and scripting potentially effective CSCL macro-scripts are challenging [3], patterns reflecting the best practices to structure collaborative learning can be useful [5]. Collaborative Learning Flow Patterns (CLFPs) define effective means to orchestrate the activity flow while implying collaboration mechanisms that can be modelled as learning design constraints (e.g., characteristics of group formation) [5]. Examples of such CLFPs are Jigsaw, Pyramid or Snowball, Thinking Aloud Pair Problem Solving (TAPPS). These patterns are governed by their pedagogical rationale (see Table 1), that are required to be preserved during the learning design in order to, not to violate the expected learning gains [3], [4]. CLFPs have been experimented and evaluated as effective to design and deploy structured CSCL activities in small scale settings for decades. Multiple works in the field have shown the feasibility of designing, authoring and deploying pattern scripts [5]-[7] in small scale, co-located learning settings.

But, research around scalable collaborative learning approaches, models and technologies for large classrooms or large learning communities is scattered across without a comprehensive body of knowledge [8]. Direct application of existing CSCL methods to large learning scenarios is challenging due to lack of scalability of such proposed methods since those are not designed to be scalable [9]. Also, due to dynamic and varied nature of large learning communities as in Massive Open Online Courses (MOOCs) where diverse learner motivations and behaviors are inherent. Various attempts have shown positive results in applying CSCL in large classes using audience response systems [10] or shared resources like display devices [11]. Yet, such studies are either much contextualized to specific learning scenarios or lack in structuring orchestration following CLFPs. Therefore within this paper, we try to identify and understand potential flow patterns for scalability and related concerns to be taken into account when applying collaborative learning in large, diverse learner communities.

We studied and analyzed three commonly used flow patterns (Pyramid, a.k.a. Snowball), Jigsaw and Thinking Aloud Pair Problem Solving (a.k.a. TAPPS) CLFPs [4] (see Table 1) in small-scale settings and present a detailed analysis of making patterns adaptable to suit large learner communities. The methodology followed in the paper is analytical; more precisely, each pattern is analyzed considering four dimensions, to inspect the level of pedagogical appealing within such learning contexts, how far it is scalable from both student and practitioner perspectives, how it is MOOC-suitable considering much diverse and unpredictable nature of MOOCs, and finally how collaborations can 
be made further meaningful. Moreover, series of potential use cases are synthesized, expressing the applicability of CLFPs to large learner communities as a key result derived from the analytical study. Finally an accumulated discussion based on above key results and derived work from the analytical study shows possible future research avenues based on this study.

Table 1. Jigsaw, Pyramid, TAPPS pattern descriptions [4]

\begin{tabular}{|c|c|c|}
\hline Jigsaw & yramid & $\begin{array}{l}\text { Thinking Aloud Pair Problem } \\
\text { Solving (TAPPS) }\end{array}$ \\
\hline $\begin{array}{l}\text { Relates to a situation where } \\
\text { several small groups of students } \\
\text { ('Jigsaw' groups), each trying to } \\
\text { solve a complex problem that can } \\
\text { be divided into sub-problems. } \\
\text { Each group participant studies one } \\
\text { sub-problem individually. Then } \\
\text { participants from different Jigsaw } \\
\text { groups, who studied similar sub } \\
\text { problems, meet in temporary } \\
\text { 'Expert' groups to exchange ideas } \\
\text { about their common sub-problems. } \\
\text { Finally, participants return back to } \\
\text { their Jigsaw groups to share } \\
\text { knowledge and solve the global } \\
\text { problem. Following educational } \\
\text { benefits might be promoted via this } \\
\text { pattern. } \\
\text { - To promote the feeling that group } \\
\text { members need each other to } \\
\text { succeed (positive } \\
\text { interdependence) } \\
\text { - To foster discussion in order to } \\
\text { construct students' knowledge } \\
\text { - To ensure that students must } \\
\text { contribute fairly (individual } \\
\text { accountability) }\end{array}$ & $\begin{array}{l}\text { Starts individually or forming initial } \\
\text { small groups (usually pairs) to } \\
\text { study a common problem and } \\
\text { propose initial solutions. Then, } \\
\text { students are grouped into small } \\
\text { groups to compare and discuss } \\
\text { their proposals and, finally, } \\
\text { propose a new shared solution } \\
\text { from the group. Students are } \\
\text { guided so that the groups join with } \\
\text { new groups to form larger groups } \\
\text { in order to generate new agreed } \\
\text { proposals. Likewise, discussions } \\
\text { and group accumulating will iterate } \\
\text { till the whole group reaches upon a } \\
\text { global consensus. Following } \\
\text { educational benefits might be } \\
\text { promoted via this pattern. } \\
\text { - To develop negotiation skills } \\
\text { - To promote the feeling that group } \\
\text { members need each other to } \\
\text { succeed (positive } \\
\text { interdependence) } \\
\text { - To foster discussion in order to } \\
\text { construct students' knowledge }\end{array}$ & $\begin{array}{l}\text { Students are paired and given a } \\
\text { series of problems. The two } \\
\text { students are given specific roles } \\
\text { (problem solver and listener) that } \\
\text { switch in each problem. The } \\
\text { problem solver reads the problem } \\
\text { aloud and talks through the } \\
\text { solution of the problem. The other } \\
\text { (the listener) follows all of the } \\
\text { problem solver's steps and catches } \\
\text { any errors that occur. The listener } \\
\text { may ask questions if the problem } \\
\text { solver's thought process becomes } \\
\text { unclear. The question asked, } \\
\text { however, should not guide the } \\
\text { problem solver to a solution nor } \\
\text { should they explicitly highlight a } \\
\text { specific error except to comment } \\
\text { that an error has been made. } \\
\text { Following educational benefits } \\
\text { might be promoted via this pattern. } \\
\text { - To foster discussion in order to } \\
\text { construct students' knowledge } \\
\text { - To permit students to rehearse } \\
\text { the concepts and produce a } \\
\text { deeper understanding of the } \\
\text { material } \\
\text { - To encourage analytical } \\
\text { reasoning skills } \\
\text { - To support problem solving skills }\end{array}$ \\
\hline
\end{tabular}

\section{CLFP ANALYSIS FOR THEIR POTENTIAL IMPLEMENTATION IN MOOCS}

We have inspected how different collaborative learning strategies including CLFPs could be adapted within MOOCs. Mostly face-to-face large classrooms have a static behaviour in nature whereas MOOCs are so divergent and dynamic, thus we selected MOOCs to be the threshold of the analysis. Each strategy is studied further to inspect level of pedagogical appealing, how far is it scalable from both students' as well as practitioners' perspectives, how this proposed technique is MOOCsuitable and finally how collaborations can be made further meaningful. The four parameters considered in the analysis are further explained as below.

Level of pedagogical appealing is concerned on how far a student could obtain learning gains and why it is interesting for a practitioner to implement that particular collaborative learning strategy. As scalable aspects, here we have considered the practicality and feasibility of managing the learning scenario by practitioners and also, the ability for learners also to easily engage in the activity, irrespective of massive number of participants. MOOC-suitable explains the flexibility of the strategy to adjust with the dynamic nature of MOOCs where students join and leave at different times during collaborative activities. The final parameter is how collaborations can be made further fruitful to allow more meaningful interactions by implying constraints such as grouping criteria or role allocation conditions [12]. Even currently existing collaboration strategies in MOOCs like peer assessment have been considered to normalize the analysis and assess the applicability. 


\subsection{Analysis of potential Peer-assessment CLFP implementation}

This strategy already exists in many MOOC platforms (e.g., open response assessments in edX, peerreviewed assignments in Coursera, peer review in FutureLearn). MOOC learners those who are willing to participate will take up these assessments. The MOOC platform will guide and help participants to receive peer-assessments and feedback when the assignments are submitted. Practitioners are required to design assessment rubrics providing criteria and options. Depending on the level of platform implementation, settings like number of responses per student to grade and number of peer assessments per response (minimum number of students that must grade each response) etc. are modifiable once designed. Over-dimensioning mechanism is adhered to ensure that all responses get required amount of peer responses. Further, the massive amount of assessments are handled and assigned automatically when designing the activity. Peer-assessment has been implemented in a quite flexible and pedagogically meaningful manner in MOOCs.

a) Pedagogically appealing: Every participant in the assessment will receive opportunities to evaluate other peers and provide feedback. MOOC platforms encourage giving useful constructive positive feedback and suggestions to improve promoting peer learning with guidance.

b) Scalable approaches:

- Easy managing for practitioners since they do not have to evaluate massive numbers of assignments submitted. Based on the number of participants and the criteria specified by practitioners, the resources (assessments) will be automatically allocated by the platform.

- From the perspective of students also feasible since they are allocated with small amount of assignments that is feasible to provide feedback.

c) MOOC-suitable as participants only willing to take peer assessment task are considered. Furthermore, peer-assessment is "over dimensioning" and used as a part of grading (penalty) or a condition to be graded. Since learners who do not participate are not considered, minimal effect for a participant to not to receive feedback.

d) Collaborations can be made further fruitful by allocating assignments according to individual's interests or expertise. Top scoring responses can be made visible globally with feedback (as in edX) or responses and feedback could be shared with all participants as either forum posts or using a different public URL (as in Coursera) or a new discussion can be emerged among the reviewer and the author once feedback is submitted (as in FutureLearn).

\subsection{Analysis of potential Pyramid CLFP implementation}

MOOC participants willing to participate in this activity will access. A possible instantiation of Pyramid flow following a "discussion bus" approach [9] is suitable, where participants individually propose a solution which then will be shared with peers' solutions in small groups. They would discuss in the local forum and agree on a common solution to be posted to the next level of the collaboration by voting according to their preference. Iteratively, they are required to discuss and vote in much larger groups. Likewise, groups will grow larger; thus the collaborations too, till only a few set of final solutions will be remaining. Solution(s) having highest votes are posted in the global forum to be seen by all participants (or even a highest reviewed response is valid) and to be addressed by the MOOC facilitators.

a) Pedagogically appealing: Everybody has the equal opportunity to express and discuss their ideas (individual accountability), social interactions, negotiation, accumulated consensus and mutual agreement with reasoning (positive interdependence).

b) Scalable approaches:

- From practitioners' perspective, it is scalable since there will be only few final answers/solutions chosen for the global forum, irrespective of the massive number of individual participants.

- As for the learners too, this mechanism would filter out thousands of responses into few most relevant and pedagogically valid replies as a collaborative knowledge building effort [13], [14] where everyone has equal opportunity to participate and raise their voices.

c) MOOC-suitable: Only the participants interested in the Pyramid activity will participate and other MOOC participants are not being considered. Pyramids are formulated on demand 
without violating pedagogical interests and conditions like having a minimum number to initiate and interact.

d) Collaborations can be made further fruitful: Pairing and grouping can be more interesting and meaningful by applying soft constraints [12], [15] into the context. For example, when finding pairs or suitable peers elements such as previous experience or their expertise or friendship (obtained from a social network) can be used. Grouping can be homogenous to promote knowledge sharing or heterogeneous to promote richer interactions with conflict resolutions.

\subsection{Analysis of potential Jigsaw CLFP implementation}

Jigsaw activity can be enacted when a task can be divided into sub problems. Those who like to participate will only be considered as activity participants. Orchestration application will guide learners to get into Jigsaw and Expert groups in respective stages of the activity. Experts will be assigned different subtasks of a global problem (e.g., solving one aspect of a question or studying one dimension of an essay type project) with separately allocated collaboration tools like forums dedicated only for these small group discussions. In the Jigsaw stage, these experts will share their ideas/knowledge in their common forum of the Jigsaw group as peer tutoring and to build awareness. These Jigsaw discussions can be made visible globally, so that all participants can view and comment.

a) Pedagogically appealing: Every participant has the capability to actively participate in knowledge sharing and co-construction.

b) Scalable:

- From educator's perspective this idea is not a scalable approach since educators still have to deal with large numbers of Jigsaw groups. Also this situation depends on the role of the teacher played within the Jigsaw activity (e.g., individual vs. group feedback or activity monitoring only)

- From student's perspective, this can be seen as scalable when local small peer groups are considered. But not with global level Jigsaw discussions

c) MOOC-suitable: Participants can join the activity only if they are willing to proceed. Further, the pedagogical constraints of the pattern definition will be preserved as group computation will be done dynamically.

d) Collaborations can be made further fruitful: The approach can be made more interesting using different constraints to derive more meaningful groupings like "selecting experts based on their expertise derived from learner profiles" or "Jigsaw belonging to same regions/countries" or "Jigsaw groups preferring to work outside MOOC platform, even using social networks such as Facebook". Orchestration tool can also help students to locate their peers easily by suggesting list of peers belonging to their location (or this can be based on social network public details) following the same MOOC and doing the current activity.

\subsection{Analysis of potential Thinking Aloud Pair Problem Solving implementation}

MOOC participants willing to participate in the TAPPS activity would be paired using the orchestration application. Participants are paired as "listeners" and "solvers" by the TAPPS implementation tool to solve specific tasks. The implementation will guide them to continue solving the problems while listener continues commenting for solver's solutions and the roles are interchanged alternatively. Another way of implementing TAPPS is by having groups of "solvers" and "listeners" interacting in groups rather than individuals. These interactions could be either synchronous or asynchronous in which comprehensive technological support has to be provided through the platform to maintain synchronicity (e.g., online chat rooms or video conferencing, etc.)

a) Pedagogically appealing: Participants willing to take the task will take part in the assigned role to express their views and to debate and discuss. If groups of "solvers" and "listeners" are implemented, individual accountability on the discussions are not ensured, thus the actual pedagogical value gained will be doubtful from individual's perspective. 
b) Scalable:

- From teacher's perspective, this approach is not scalable since there will be massive numbers of pair discussions to handle or to monitor and provide feedback (if they're supposed to do so).

- For the students, this is scalable considering their two roles only, but this is only possible without teacher monitoring or providing feedback for the discussion/activity flow since it is impractical.

c) MOOC suitable: The implementation is challenging since the pattern definition states that the solver reads aloud and talks about the solution for the listener to follow and comment. In order to maintain a synchronous communication, comprehensive task supported environment with video conferencing or voice and chat features are needed (e.g., Tandem [16] enables synchronous communication in MOOCs). On the contrary, typical discussion boards could also be used if only asynchronous communication is valued.

d) Collaborations can be made fruitful further: Pairing could be more interesting by conditioning. For example, when finding pairs, friendship (obtained from a social network) or the location (since they can even meet up if they prefer) can be considered.

\section{USE CASES FOR POTENTIAL CLFP ORCHESTRATION IN MOOCS}

After analysing the three CLFPs for their applicability in large collaborative learning scenarios, we synthesized some sample use case scenarios to assess the usefulness and the technological viability. The analysis reveals better prospects for scalability with Pyramid (Snowball) CLFP; thus there are more use cases considering Pyramid CLFP with various possibilities. Following use cases illustrate possible practitioner - learner interactions using sample user-interfaces followed with explanations and rationalization. Each use case describes what practitioners will do during the design stage using the technologically-supported orchestration application and how students can engage with the activity.

\subsection{Use case 01 - Quick consensus Pyramid CLFP}

Design a Pyramid collaborative learning flow activity in which students rate peer responses. Practitioners become active participants only at the activity design stage and in the final stage of the Pyramid (Fig. 1). This activity can be considered as quick consensus collaborative knowledge construction [14] with everyone's engagement. A practitioner sets required design parameters for a Pyramid (e.g., levels, group size, etc.) and defines activities for each level (e.g., rating peer responses and responding to given question/s) along with any grouping criteria. Students willing to participate are the actors who access the Pyramid flow designed (Fig. 2). They will rate peer responses (local and global levels) and also access respective collaboration tools provided (e.g., response thread / forum, social network, etc.) (Fig. 3).

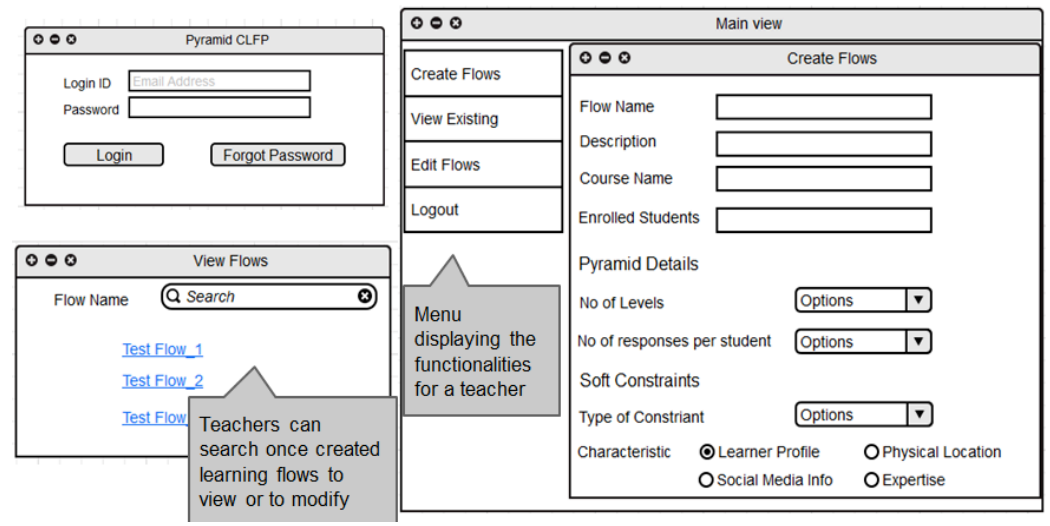

Figure 1. Pyramid case 01 interface designs for educators 
Activity : Progressive consensus for optimal solution for a given open question

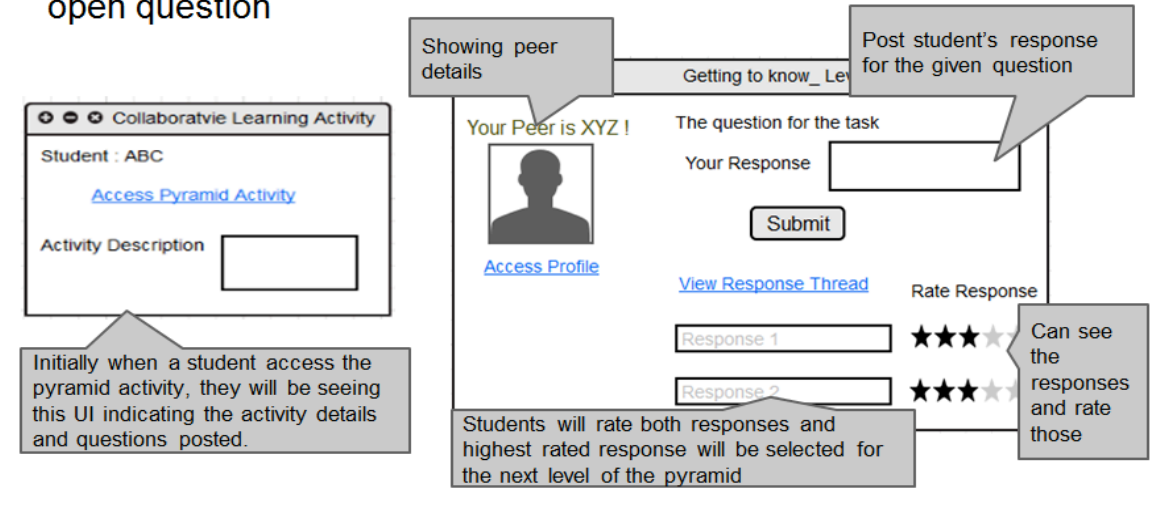

Figure 2.Pyramid case 01initial view for students
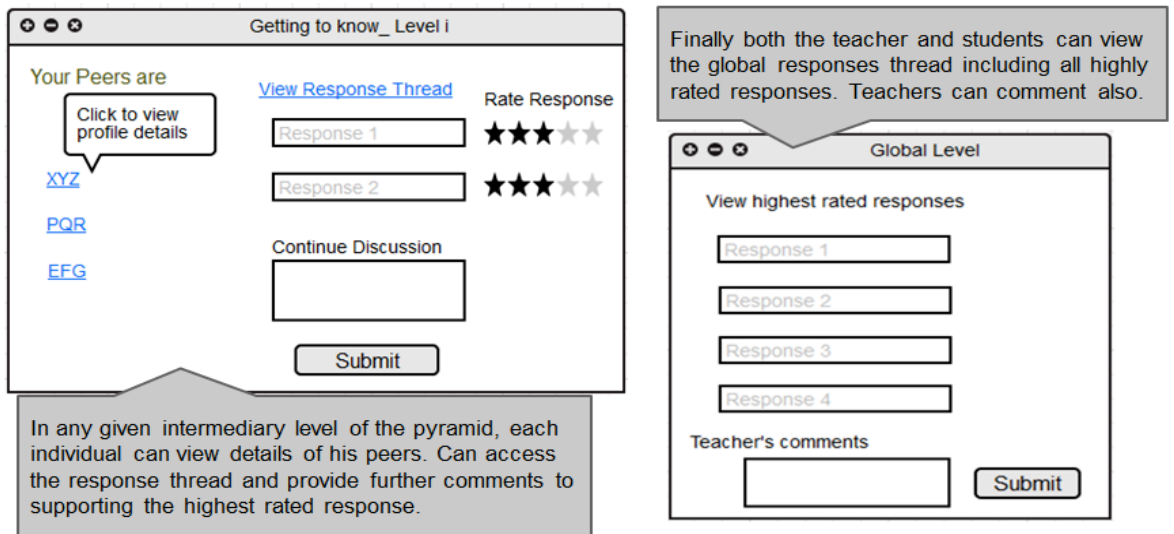

Figure 3. Pyramid case 01 intermediary interfaces for students view

\subsection{Use case 02 - Integration oriented consensus Pyramid CLFP}

Design a Pyramid collaborative learning flow activity promoting conversational theory and negotiation with positive interactions to achieve a global consensus and select the most valid question to be presented (Fig. 4). This follows integration-oriented consensus building [14] and create knowledge collaboratively. Practitioners design Pyramids by defining the levels, group sizes and other related details. Possible activity could be to define question themes for each unit to be enacted as Pyramid activities (this type of activity can be repeated with every topic). They can set pairing or group formation criteria too. Students willing to participate are the actors of this activity who will access discussion forum to negotiate the questions to be posted (Fig. 5).

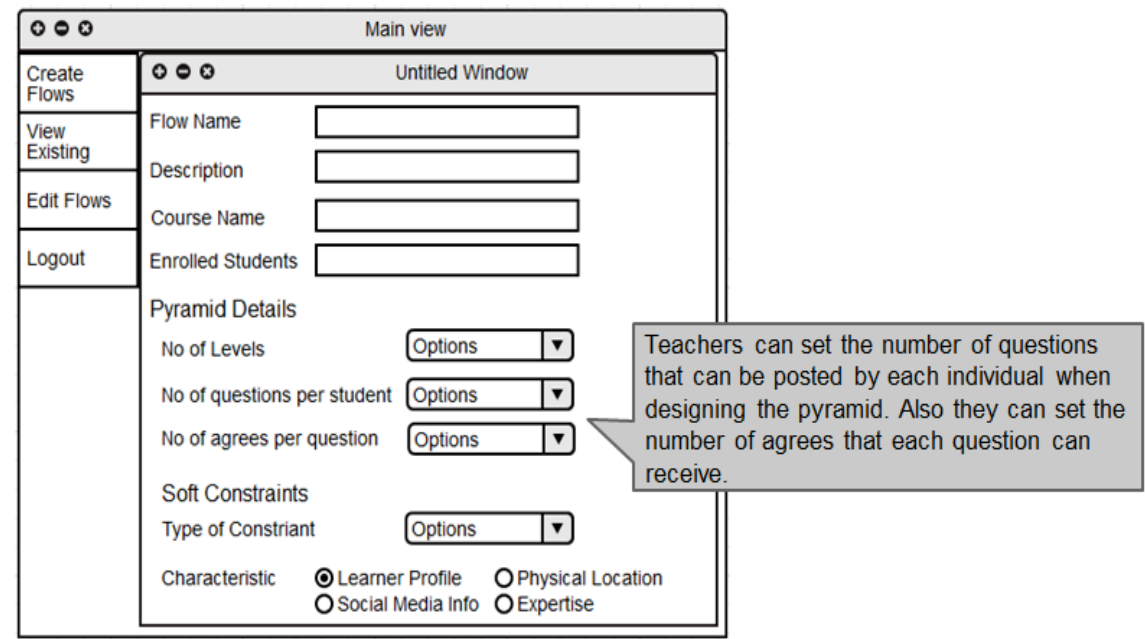

Figure 4. Pyramid case 02 interface design for educators 

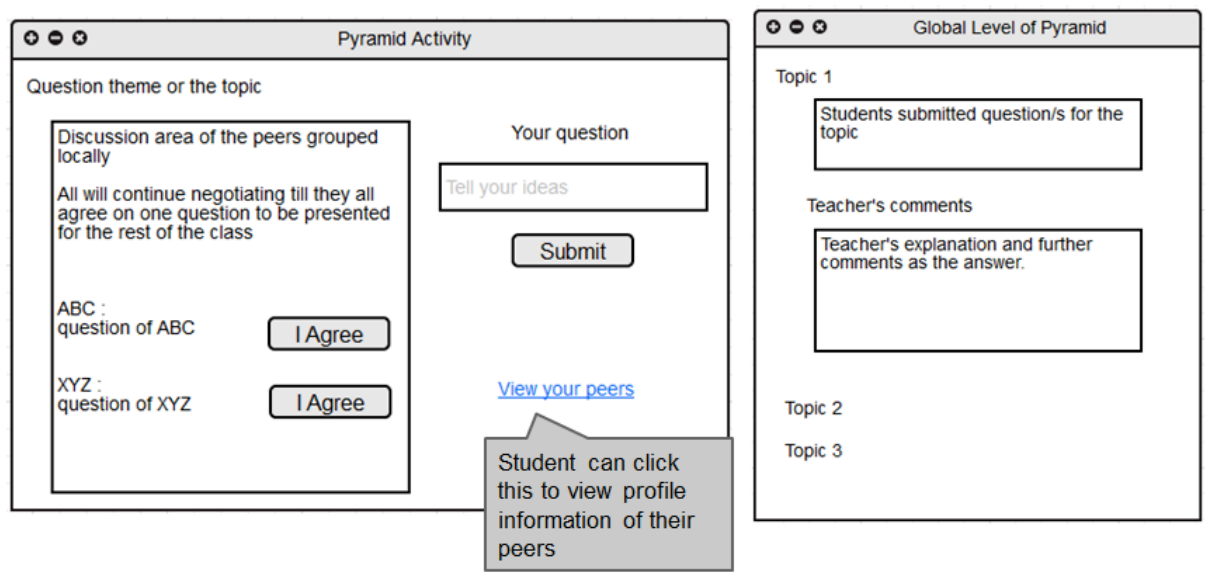

Figure 5. Pyramid case 02 interface designs for students

\subsection{Use case 03 - Conflict oriented consensus Pyramid CLFP}

Design a Pyramid collaborative learning flow enabling progressive knowledge construction via stating their willingness or conflict regarding peer's perspectives while providing individual argument (Fig. 6). This methodology of knowledge construction is conflict - oriented consensus building [14] in collaborative learning which could be enacted as a weekly basis on-going activity. As in above two cases, here also practitioners set the design parameters of the Pyramid, define the activities and grouping criteria, if any. Students willing to participate are the actors in this activity also. After initiating the activity, they will state their willingness or the conflict for the comments and provide the rationale (individual perspective) during the negotiation process. Also they will access any collaboration space provided (e.g., forum).
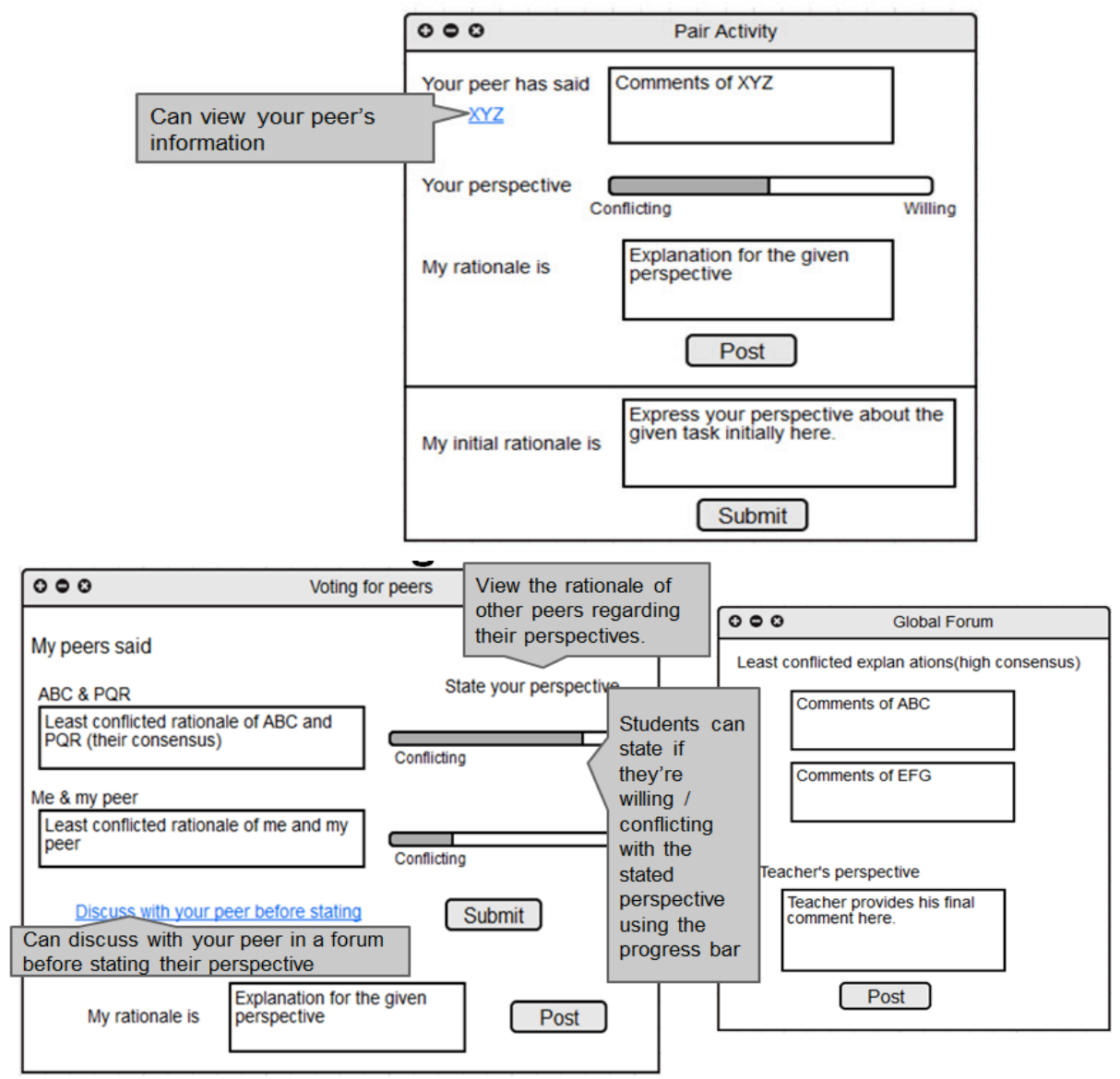

Figure 6. Pyramid case 03 interface designs for students 


\subsection{Use case 04 - Jigsaw CLFP}

The approach would be applicable and scalable only if the practitioners' involvement is less or filtered only for the higher levels, the activity can be sub divided and the pattern is complemented with a Pyramid structure to avoid lengthy forum discussions. Jigsaw promotes individual accountability [4], hence the initial levels of Jigsaw will allow students to engage more fruitfully at individual levels contributing towards the final goal while sharing conceptions and learning from the massive community. Design a Jigsaw activity to collaboratively create knowledge with peers by exchanging their specific knowledge obtained as experts to educate others (Fig. 7). Practitioners can create the sub tasks for expert groups (discussion oriented tasks on sub topics or any task that could be sub divided or even project tasks and idea generation activities). Moreover, they can specify any grouping condition accordingly. To engage in the Jigsaw activity, students willing to participate can access it (Fig. 8). They will have access to respective Expert and Jigsaw groups and discuss on the captured knowledge in forums or in other collaborative learning tools. Finally they will access global forum to view responses and comment.

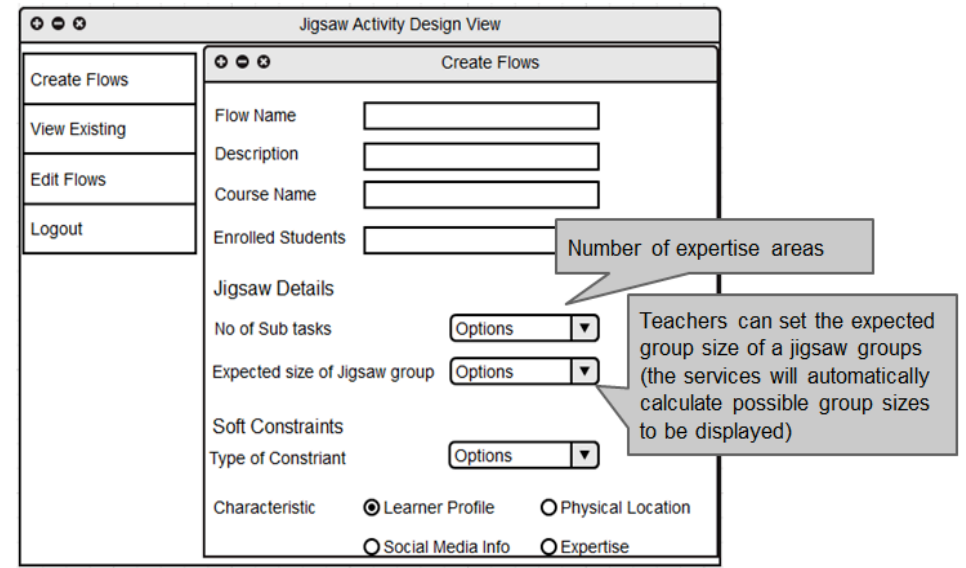

Figure 7. Educator interface for a Jigsaw activity design

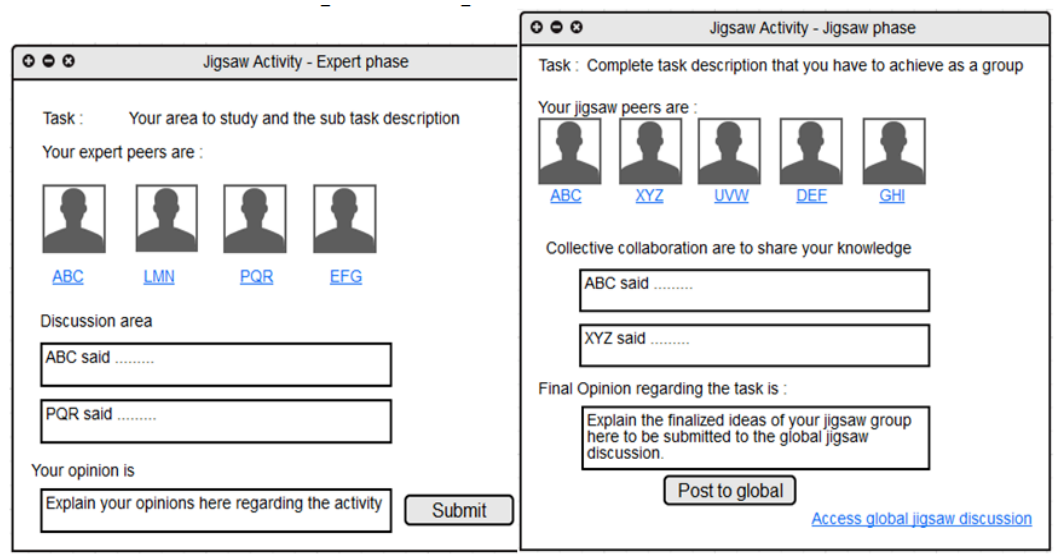

Figure 8. Student interfaces for Jigsaw activity

\subsection{Use case 05 - TAPPS CLFP}

To overcome the limitations of this proposed use case and to introduce diverse types of activities using TAPPS, a comprehensive technological environment should be presented as highlighted in the above analysis section in order to maintain "aloud" feature in the pattern precisely (e.g., provide synchronous communications mechanisms, etc.). If the activity is implemented as asynchronous, following suggestion will be suitable. Further, to make the pattern scalable from practitioners' point of view, it can be complemented with a Pyramid in which the educator becomes a mere observer during the activity enactment. Practitioners can design a series of questions as the task with interchanging problem solver/ listener roles enabling knowledge construction through conversing among themselves. To engage in the TAPPS activity, students willing to participate will access the activity. They will identify their roles and continue with the discussion by accessing required collaboration tools (e.g., video conferencing or chat tools like hangout or a forum) (Fig. 9). 


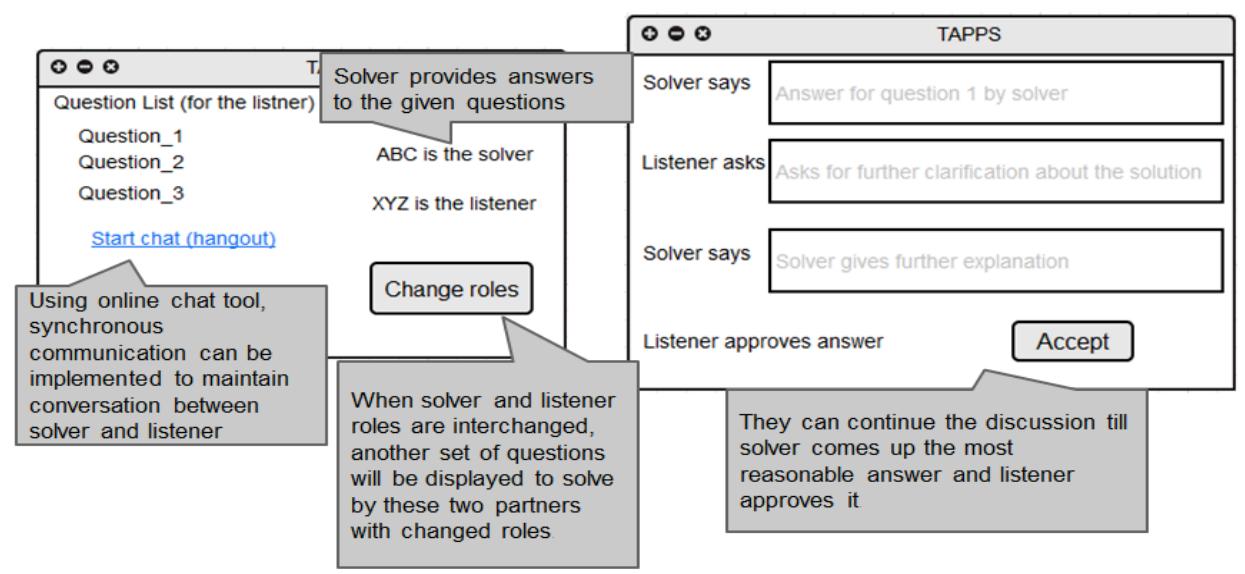

Figure 9. Interface design for students in a TAPPS activity

\section{DISCUSSION: THE PYRAMIDAPP TOOL}

We considered three commonly used flow patterns (Jigsaw, Pyramid and TAPPS) and analyzed those considering four parameters to assess pattern applicability in large learning scenarios. The analysis exposed possibilities and challenges that CLFPs pose within such implementations. In particular, Pyramid collaborative learning flow pattern is identified with more potential for scalability. Consequently, Pyramid pattern is instantiated as a scalable model and technologically implemented as a fully-fledged application called "PyramidApp", composed of both Pyramid flow authoring and enactment aspects. PyramidApp is a web-based, openly accessible tool implemented using technologies like JavaScript to serve the front-end, PHP and MySQL at the back-end. Several rounds of evaluation studies, varying from secondary school level to Masters' level have been used in the process and three experimental studies and obtained results have been published in [17]. Another two evaluation studies using PyramidApp in the context of a MOOC and the related results are reported in [18]. With accumulating collaborations, while being scalable and preserving dynamism in the flow, PyramidApp received positive perceptions from both practitioners and students.

\section{CONCLUSIONS}

Collaborative learning is the notion of knowledge creation by means of effective interactions and CLFPs are well-known best practices that support to orchestrate collaborative learning activities to generate potential fruitful interactions. Yet, patterns have been experimented and evaluated mainly in small learning scenarios and how those can be applied to large and dynamic learning contexts is under-explored. In this work, we present an analysis of three commonly used patterns (Pyramid, Jigsaw and TAPPS CLFPs) in small-scale settings in order to be adaptable in large learner communities. This analytical study reveals details of each pattern in four different dimensions such as the level of pedagogical appealing when applied on large learning contexts, scalability from both student's and practitioner's perspectives, MOOC-suitability considering unpredictable dynamic nature of MOOCs, and how collaborations can be made further meaningful. After studying the results of the analysis, we synthesized a series of potential use cases along with initial user interface designs, expressing the rationale. Among all possibilities, Pyramid pattern showed interesting scalable scenarios that drove us to particularize the pattern and technologically support the authoring, enactment and orchestration via PyramidApp. This particularization is one example of scalable CSCL approaches; yet above analytical study reveals more suggestions to design and implement scalable CSCL orchestration technologies inspired by the use cases synthesized.

\section{ACKNOWLEDGEMENTS}

This work has been partially funded by the Spanish Ministry of Economy and Competitiveness under RESET (TIN2014-53199-C3-3-R), the Maria de Maeztu Units of Excellence Programme (MDM-20150502) and RecerCaixa (CoT project). 


\section{REFERENCES}

[1] P. Dillenbourg, "What do you mean by 'collaborative learning'?," in Collaborative Learning: Cognitive and Computational approaches (P. Dillenbourg, Eds.), vol. 1, no. 6, pp. 1-15, 1999.

[2] P. Dillenbourg and P. Jermann, "Technology for Classroom Orchestration," in New Science of Learning: Cognition, Computers and Collaboration in Education (M.S. Khine and I. Saleh, Eds.), pp. 45-52, 2010.

[3] P. Dillenbourg and P. Tchounikine, "Flexibility in macro-scripts for CSCL," Journal of Computer Assisted Learning, 23, pp. 1-13, 2007.

[4] D. Hernández-Leo, J. I. Asensio-Pérez, Y. Dimitriadis, and E. D. Villasclaras-Fernández, "Generating cscl scripts," in Technology-Enhanced Learning, Design Patterns and Pattern Languages (P. Goodyear and S. Retalis, Eds.), pp. 49-64, Rotterdam: Sense Publishers, 2010.

[5] D. Hernández-Leo, E. D. Villasclaras-Fernández, J. I. Asensio-Pérez, Y. Dimitriadis, I. M. JorrínAbellán, I. Ruiz-Requies, and B. Rubia-Avi, "COLLAGE: A collaborative Learning Design editor based on patterns," Educational Technology and Society, vol. 9, no. 1, pp. 58-71, 2006.

[6] M. Pérez-Sanagustín, J. Burgos, D. Hernández-Leo, and J. Blat, "CLFP intrinsic constraintsbased group management of blended learning situations," Studies in Computational Inteligence, vol. 350, pp. 115-133, 2011.

[7] M. J. Rodríguez-Triana, "Linking scripting and monitoring in blended CSCL Linking scripting \& monitoring support in blended CSCL scenarios," Dr. Diss. University of Valladolid, Spain, 2014.

[8] K. Manathunga and D. Hernández-Leo, "Has research on collaborative learning technologies addressed massiveness? A literature review," Educational Technology and Society, vol. 18, no. 4, pp. 357-370, 2015.

[9] R. Ferguson and M. Sharples, "Innovative Pedagogy at Massive Scale : Teaching and Learning in MOOCs," in Open Learning and Teaching in Educational Communities, proceedings of 9th European Conference on Technology Enhanced Learning, vol. 8719, pp. 98-111, 2014.

[10] C. F. Herreid, "Clicker' Cases: Introducing Case Study Teaching Into Large Classrooms," Journal of College Science Teaching, vol. 36, no. 2, pp. 43-47, 2006.

[11] E. Szewkis, M. Nussbaum, T. Rosen, J. Abalos, F. Denardin, D. Caballero, A. Tagle, and C. Alcoholado, "Collaboration within large groups in the classroom," International Journal of Computer-supported Collaborative Learning, vol. 6, no. 4, pp. 561-575, 2011.

[12] K. Manathunga and D. Hernández-Leo, "A Multiple Constraints Framework for Collaborative Learning Flow Orchestration," in Advances in Web-Based Learning - ICWL 2016, vol. 10013, pp. 225-235, 2016.

[13] M. Scardamalia and C. Bereiter, "Knowledge Building: Theory, Pedagogy, and Technology," Cambridge Handook of Learning Sciences, pp. 97-118, 2006.

[14] A. Weinberger and F. Fischer, "A framework to analyze argumentative knowledge construction in computer-supported collaborative learning," Computers and Education, vol. 46, no. 1, pp. $71-$ 95, 2006.

[15] P. Dillenbourg, "Over-scripting CSCL: The risks of blending collaborative learning with instructional design," in Three worlds of CSCL: Can we Support CSCL? (P. A. Kirschner, Eds.), pp. 61-91, 2002.

[16] M. C. Fondo Garcia, "Synchronous Tandem Language Learning in a MOOC Context: A Study on Task Design and Learner Performance," Research-publishing.net, 2016.

[17] K. Manathunga and D. Hernández-Leo, "PyramidApp: Scalable Method Enabling Collaboration in the Classroom," in Adaptive and adaptable learning: 11th European Conference on Technology Enhanced Learning, vol. 9891, pp. 422-427, 2016.

[18] K. Manathunga, D. Hernández-Leo, and M. Sharples, "A Social Learning Space Grid for MOOCs : Exploring a FutureLearn Case," in Fifth European MOOCs Stakeholders Summit (to appear in Springer LNCS), 2017. 